\title{
Scaled models, scaled frequencies, and model fitting (Research Note)
}

\author{
Ian W. Roxburgh
}

\begin{abstract}
Astronomy Unit, Queen Mary University of London, Mile End Road, London E1 4NS, UK e-mail: I.W.Roxburgh@qmul.ac.uk
\end{abstract}

Received 24 September 2015 / Accepted 3 November 2015

\section{ABSTRACT}

\begin{abstract}
I show that given a model star of mass $M$, radius $R$, and density profile $\rho(x)[x=r / R]$, there exists a two parameter family of models with masses $M_{k}$, radii $R_{k}$, density profile $\rho_{k}(x)=\lambda \rho(x)$ and frequencies $v_{n \ell}^{k}=\lambda^{1 / 2} v_{n \ell}$, where $\lambda, R_{k} / R_{\mathrm{A}}$ are scaling factors. These models have different internal structures, but all have the same value of separation ratios calculated at given radial orders $n$, and all exactly satisfy a frequency matching algorithm with an offset function determined as part of the fitting procedure. But they do not satisfy ratio matching at given frequencies nor phase shift matching. This illustrates that erroneous results may be obtained when model fitting with ratios at given $n$ values or frequency matching. I give examples from scaled models and from non scaled evolutionary models.
\end{abstract}

Key words. stars: oscillations - asteroseismology - stars: interiors - methods: analytical - methods: numerical

\section{Scaled models}

Consider a reference Model A with mass $M_{\mathrm{A}}$, radius $R_{\mathrm{A}}$, and density profile $\rho_{\mathrm{A}}\left(x_{\mathrm{A}}\right)$, where $x_{\mathrm{A}}=r / R_{\mathrm{A}}$ is the fractional radius, and a scaled Model $\mathrm{B}$ of mass $M_{\mathrm{B}}$, radius $R_{\mathrm{B}}$ density profile $\rho_{\mathrm{B}}\left(x_{\mathrm{B}}\right)=\lambda \rho_{\mathrm{A}}\left(x_{\mathrm{A}}\right)$ where $x_{\mathrm{B}}=r / R_{\mathrm{B}}$ and $\lambda$ is a scaling factor. Both models satisfy the hydrostatic and mass equations

$\frac{\mathrm{d} P}{\mathrm{~d} r}=-\frac{G M(r)}{r^{2}} \rho(r), \quad \frac{\mathrm{d} M(r)}{\mathrm{d} r}=4 \pi r^{2} \rho(r)$.

The mass variable $M(x)$ of the 2 models are given by

$\frac{M_{\mathrm{A}}(x)}{4 \pi R_{\mathrm{A}}^{3}}=\int_{0}^{x} \rho_{\mathrm{A}}(x) x^{2} \mathrm{~d} x, \quad \frac{M_{\mathrm{B}}(x)}{4 \pi R_{\mathrm{B}}^{3}}=\int_{0}^{x} \rho_{\mathrm{B}}(x) x^{2} \mathrm{~d} x$

and as $\rho_{\mathrm{B}}(x)=\lambda \rho_{\mathrm{A}}(x)$ it follows that

$M_{\mathrm{B}}(x)=\lambda\left(\frac{R_{\mathrm{B}}}{R_{\mathrm{A}}}\right)^{3} M_{\mathrm{A}}(x), \quad M_{\mathrm{B}}=\lambda\left(\frac{R_{\mathrm{B}}}{R_{\mathrm{A}}}\right)^{3} M_{\mathrm{A}}$.

The pressures $P_{\mathrm{A}}(x), P_{\mathrm{B}}(x)$ satisfy

$\frac{\mathrm{d} P_{\mathrm{A}}(x)}{\mathrm{d} x}=-\frac{G M_{\mathrm{A}}(x)}{R_{\mathrm{A}} x^{2}} \rho_{\mathrm{A}}(x), \quad \frac{\mathrm{d} P_{\mathrm{B}}(x)}{\mathrm{d} x}=-\frac{G M_{\mathrm{B}}(x)}{R_{\mathrm{B}} x^{2}} \rho_{\mathrm{B}}(x)$

SO

$\frac{\mathrm{d} P_{\mathrm{B}}(x)}{\mathrm{d} P_{\mathrm{A}}(x)}=\frac{R_{\mathrm{A}}}{R_{\mathrm{B}}} \frac{M_{\mathrm{B}}(x)}{M_{\mathrm{A}}(x)} \frac{\rho_{\mathrm{B}}(x)}{\rho_{\mathrm{A}}(x)}, \quad$ so $P_{\mathrm{B}}(x)=\lambda^{2}\left(\frac{R_{\mathrm{B}}}{R_{\mathrm{A}}}\right)^{2} P_{\mathrm{A}}(x)$

and

$g_{\mathrm{B}}(x)=\frac{G M_{\mathrm{B}}(x)}{R_{\mathrm{B}}^{2} x^{2}}=\lambda\left(\frac{R_{\mathrm{B}}}{R_{\mathrm{A}}}\right)^{3} \frac{G M_{\mathrm{A}}(x)}{R_{\mathrm{B}}^{2} x^{2}}=\lambda\left(\frac{R_{\mathrm{B}}}{R_{\mathrm{A}}}\right) g_{\mathrm{A}}(x)$.

\section{Scaled frequencies}

For simplicity of presentation I first consider radial $(\ell=0)$ oscillations where the equations governing the Lagrangian pressure perturbation $\delta p$, and radial displacement $\xi$, with angular frequency $\omega$, can be written as (cf. Roxburgh 2008)

$\frac{\mathrm{d} \xi}{\mathrm{d} r}=-\frac{2}{r} \xi-\frac{1}{\Gamma_{1} p} \delta p$

$\frac{\mathrm{d} \delta p}{\mathrm{~d} r}=\omega^{2} \rho \xi+\frac{4 g \rho \xi}{r}$

Defining $y=\xi / R$ and $x=r / R$ then for the scaled Model B

(2) $\frac{\mathrm{d} y}{\mathrm{~d} x}=-\frac{2}{x} y-\frac{1}{\Gamma_{1}^{\mathrm{B}}} \frac{\delta p_{\mathrm{B}}}{p_{\mathrm{B}}}$

$\frac{\mathrm{d} \delta p_{\mathrm{B}}}{\mathrm{d} x}=\omega_{\mathrm{B}}^{2} \rho R_{\mathrm{B}}^{2} y+\frac{4 g_{\mathrm{B}} R_{\mathrm{B}} \rho_{\mathrm{B}} y}{x}$.

Taking $\Gamma_{1}^{\mathrm{B}}(x)=\Gamma_{1}^{\mathrm{A}}(x)$, (a good approximation in the fully ionised interior) then, since both $y$ and $\delta p / p$ are invariant under the scaling, Eq. (9) is invariant under the scaling from the reference model. Substituting the scaling relations

$\rho_{\mathrm{B}}=\lambda \rho_{\mathrm{A}}, \quad \delta p_{\mathrm{B}}=\lambda^{2}\left(\frac{R_{\mathrm{B}}}{R_{\mathrm{A}}}\right)^{2} \delta p_{\mathrm{A}}, \quad g_{\mathrm{B}}=\lambda\left(\frac{R_{\mathrm{B}}}{R_{\mathrm{A}}}\right) g_{\mathrm{A}}$

into Eq. (10) then, with a little manipulation, one obtains

$\frac{\mathrm{d} \delta p_{\mathrm{A}}}{\mathrm{d} x}=\omega_{\mathrm{A}}^{2} \rho R_{\mathrm{A}}^{2} y+\frac{4 g_{\mathrm{A}} R_{\mathrm{A}} \rho_{\mathrm{A}} y}{x}, \quad$ where $\omega_{\mathrm{A}}^{2}=\frac{1}{\lambda} \omega_{\mathrm{B}}^{2}$.

To summarise, given $\lambda, R_{\mathrm{B}}$ then the scaled models satisfy

$\frac{M_{\mathrm{B}}}{R_{\mathrm{B}}^{3}}=\lambda \frac{M_{\mathrm{A}}}{R_{\mathrm{A}}^{3}}, \quad \rho_{\mathrm{B}}(x)=\lambda \rho_{\mathrm{A}}(x), \quad P_{\mathrm{B}}(x)=\lambda^{2}\left(\frac{R_{\mathrm{B}}}{R_{\mathrm{A}}}\right)^{2} P_{\mathrm{A}}(x)$

$\omega_{\mathrm{B}}^{2}=\lambda \omega_{\mathrm{A}}^{2}$ so $v_{n \ell}^{\mathrm{B}}=\lambda^{1 / 2} v_{n \ell}^{\mathrm{A}}$ 

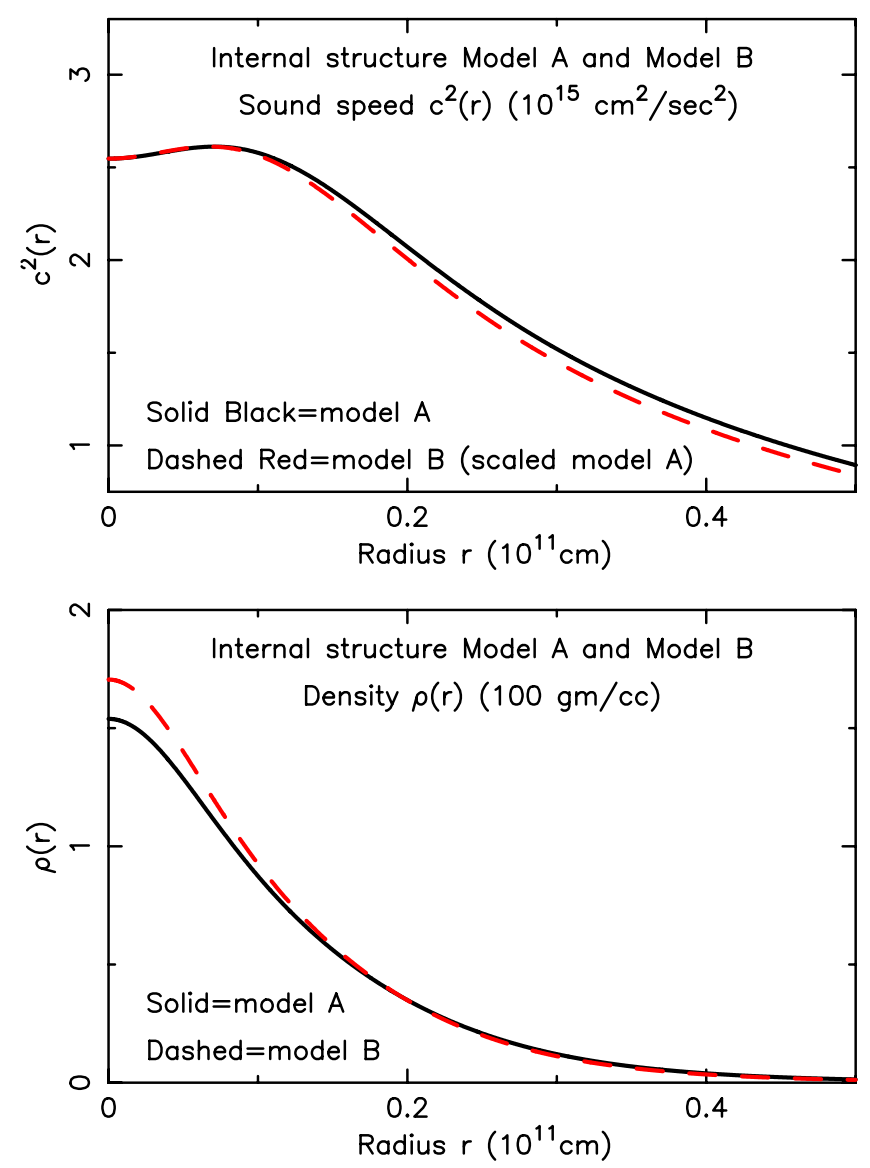

Fig. 1. Internal structure of Model A and the scaled model, Model B. Top panel: variation of $c^{2}(r)$ for Models A and B; Bottom panel: density $\rho(r)$ in the two models.

where I have anticipated the result (derived in the Appendix) that these relations hold for modes any angular degree $\ell$.

If $\lambda=1$ then one recovers the well known result that the frequencies $v_{n \ell}$ are invariant under a scaling with $M / R^{3}$ constant.

\section{An example}

To illustrate these results I take for the reference Model A a solar model based on Model S of Christensen-Dalsagaard et al. (1996), the scaled Model $\mathrm{B}$ has a radius $R_{\mathrm{B}}=0.95 R_{\mathrm{A}}$ and $\lambda=\left(R_{\mathrm{A}} / R_{\mathrm{B}}\right)^{2}=1.108 \ldots$, so $M_{\mathrm{B}}=0.95 M_{\mathrm{A}}$ and the models have the same $c^{2}(x)=\Gamma_{1} P / \rho$ (cf. Eqs. (13)); but they do not have the same $c^{2}(r)$ as a function of radius $r$. The run of density $\rho(r)$ and $c^{2}(r)$ for the two models are shown in Fig. 1, clearly Model B does not have the same interior structure as Model A.

\section{Model fitting with separation ratios}

Since the frequencies scale like $\lambda^{1 / 2}$ the frequencies of Model B are not equal to the frequencies of Model A. Nevertheless the ratios of small to large separations, which is often used in surface layer independent model fitting, are identical when calculated at the same radial order $n$. This is illustrated in the top panel of Fig. 2 for the separations $r_{02}(n)$ defined as (cf. Roxburgh \& Vorontsov 2003a,b)

$r_{02}(n)=\frac{v_{n, 0}-v_{n-1,2}}{v_{n, 1}-v_{n-1,1}}$
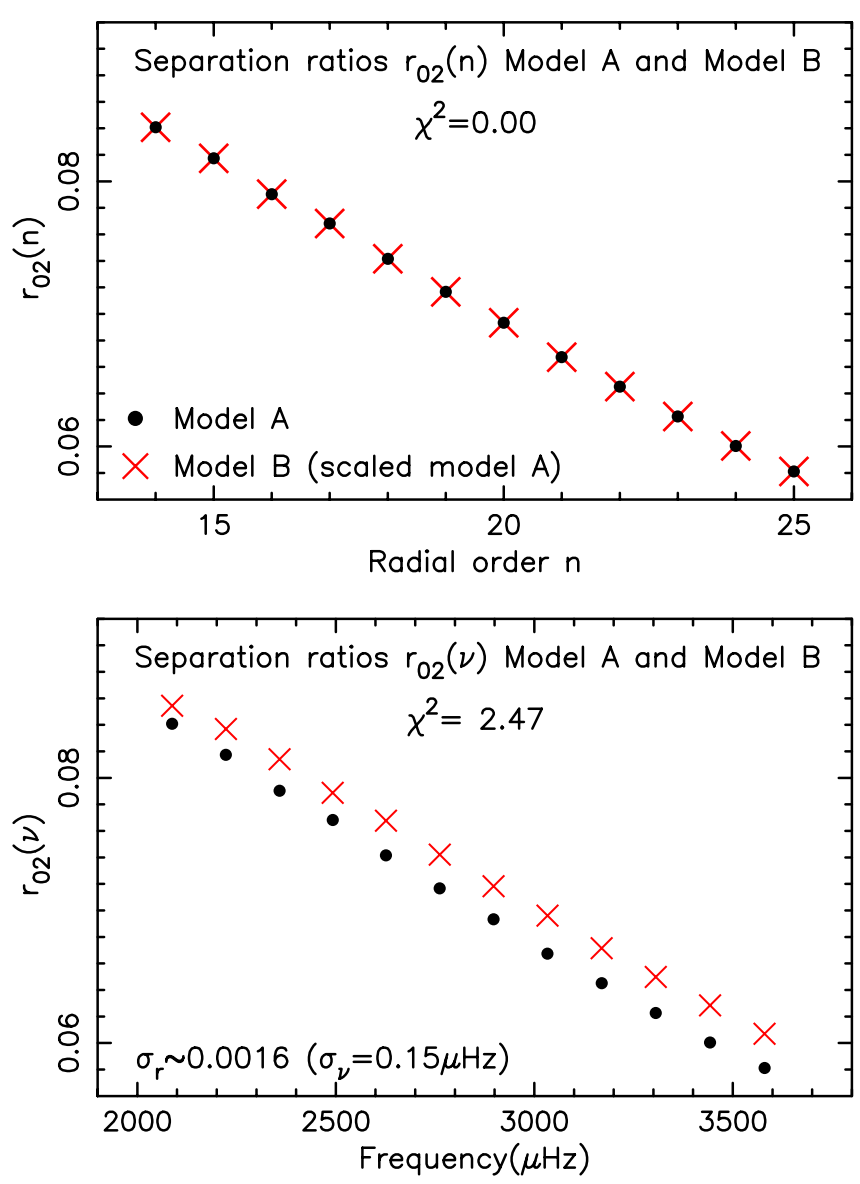

Fig. 2. Comparison of separation ratios $r_{02}$ of Model A and the scaled Model B. Top panel: comparison at the same $n$ where the fit is exact; bottom panel: comparison at the same frequencies, which is equivalent to comparing inner phase shift differences; were the two models to have the same interior structure agree these would be the same.

The frequency set for Model A has 13 frequencies for $\ell=0,1,2$ in the range $2000-3700 \mu \mathrm{Hz}$ with error estimate of $0.15 \mu \mathrm{Hz}$

The separation ratios are one way of determining the inner phase shift differences $\delta_{2}(v)-\delta_{0}(v)$ as a function of frequency, which is determined by the inner structure of the star $\left\{\rho(r), c^{2}(r)\right\}$ almost independent of the structure of the outer layers, and comparing ratios at the same $n$ is not, in general, the same as comparing at the same frequency (cf. Roxburgh \& Vorontsov 2013). To test whether a model and star have the same interior structure, and hence the same $\delta_{2}(v)-\delta_{0}(v)$ one has to interpolate in the model values of $r_{02}(n)$ to determine the values of $\delta_{2}(v)-\delta_{0}(v)$ at the same frequencies. The result of doing this for Model B is shown if the bottom panel of Fig. 2. They ratios do not agree - as one would expect since Model B does not have the same inner structure as Model A. With an error estimate of $0.15 \mu \mathrm{Hz}$ on the frequencies of Model $\mathrm{A}$, the resulting $\chi^{2}$ (per degree of freedom) of the fit is 2.47 . The probability of such a large value of $\chi^{2}$ being due to a chance realisation of errors is 0.003 .

The reason for the exact fit of the $r_{02}(n)$ ratios is quite simply that all frequencies of Model B are the same multiple of those of Model A, $v_{n \ell}^{\mathrm{B}}=\lambda^{1 / 2} v_{n \ell}^{\mathrm{A}}$, and the ratios as defined in Eq. (17) are independent of $\lambda$

This illustrates the importance of comparing ratios interpolated to the same frequencies when searching for a model fit to an observed star (cf. Roxburgh \& Vorontsov 2013). 
Table 1. Models A1 and B1.

\begin{tabular}{ccccccccc}
\hline \hline Model & $M / M_{\odot}$ & $L / L_{\odot}$ & $R / R_{\odot}$ & $X H$ & $Z$ & $\alpha$ & $X_{\mathrm{c}}$ & age 9 \\
\hline A1 & 1.15 & 1.87 & 1.25 & 0.72 & 0.021 & 1.80 & 0.096 & 4.601 \\
B1 & 1.12 & 1.90 & 1.20 & 0.70 & 0.020 & 2.00 & 0.096 & 4.128 \\
\hline
\end{tabular}

\section{Model fitting by comparing frequencies}

Shortly after Kjeldsen et al. (2008) suggested that one could model the unknown effects of the stellar surface layers by scaling the solar offset to other stars, I suggested (erroneously!) that one could treat the offset as unknown and solve for it as part of a model fitting procedure (e.g. Roxburgh 2009). I subsequently realised that such a technique suffered from the same problems as comparing separation ratios at the same $n$ values rather than at the same frequencies, as I now demonstrate.

In this algorithm one compares observed and model frequencies after having subtracted off an $\ell$ independent function of frequency $\mathcal{F}(v)$ from the frequency differences $v_{n \ell}^{\mathrm{A}}-v_{n \ell}^{\mathrm{B}}$, defining the goodness of fit $\chi^{2}$ as

$\chi^{2}=\frac{1}{N-M} \sum_{1}^{N}\left(\frac{v_{n \ell}^{\mathrm{A}}-v_{n \ell}^{\mathrm{B}}-\mathcal{F}\left(v^{\mathrm{A}}\right)}{\sigma_{n \ell}^{\mathrm{A}}}\right)^{2}$

where $\sigma_{n \ell}^{\mathrm{A}}$ are the error estimates on $v_{n \ell}^{\mathrm{A}}$ (taken to be the observed star), $N$ is the number of frequencies and $M$ the number of parameters in $\mathcal{F}(v)$ which are adjusted to minimise $\chi^{2}(M \leq$ number of $\ell=0$ modes). The difference between this and the Kjeldsen et al. (2008) method is that in their method $\mathcal{F}(v)$ is assumed known in terms of the frequencies $v_{n \ell}^{\mathrm{A}}$ and $v_{n \ell}^{\mathrm{B}}$, whereas here it is to be determined as part of the fitting process.

Since for the scaled models $v_{n \ell}^{\mathrm{B}}=\lambda^{1 / 2} v_{n \ell}^{\mathrm{A}}$ the $\chi^{2}$ for any value of $\lambda$ is reduced to zero on taking $\mathcal{F}(v)=\left[1-\lambda^{1 / 2}\right] v$, implying that all such models fit the interior structure of Model A, which is not the case.

\section{A non scaled example}

It might be thought that the results in the previous two sections are special since I took a scaling that gives $c_{\mathrm{B}}^{2}(x)=c_{\mathrm{A}}^{2}(x)$ but this is not the case. Any scaled model with will give equality of the ratios at the same $n$ values but not at the same frequencies if $\lambda \neq 1$, independent of the radius of Model $\mathrm{B}$, which could be the same as that of Model A.

But perhaps a more convincing case is to compare two separate models which are not obtained by scaling and do not necessarily have the same variation of $\Gamma_{1}(x)$. Such an example is afforded by comparing 2 models, A1 and B1, constructed using my evolution code (Roxburgh 2008), with GS98 relative abundances (Grevesse \& Sauval 1998), OPAL opacities (Iglesias \& Rogers 1996) supplemented by Wichita opacities at low temperatures (Ferguson et al. 2005), the EOS5 equation of state (Rogers \& Nayfonov 2002), NACRE reaction rates (Angulo et al. 1999), the mixing length model of convection and an Eddington atmosphere. The properties of the two models are given in Table 1 where $X H$ is the initial hydrogen abundance, $Z$ the heavy element abundance, $\alpha$ the mixing length parameter, $X_{\mathrm{c}}$ the central hydrogen abundance and age 9 the age in units of $10^{9} \mathrm{yr}$. The radius and luminosity of Model B1 differ from those of Model A by less than $5 \%$. The frequency set of Model A1 (taken as the observed star) has $14 \ell=0,1,2$ modes, and again I take an error estimate of $0.15 \mu \mathrm{Hz}$ on the frequencies.
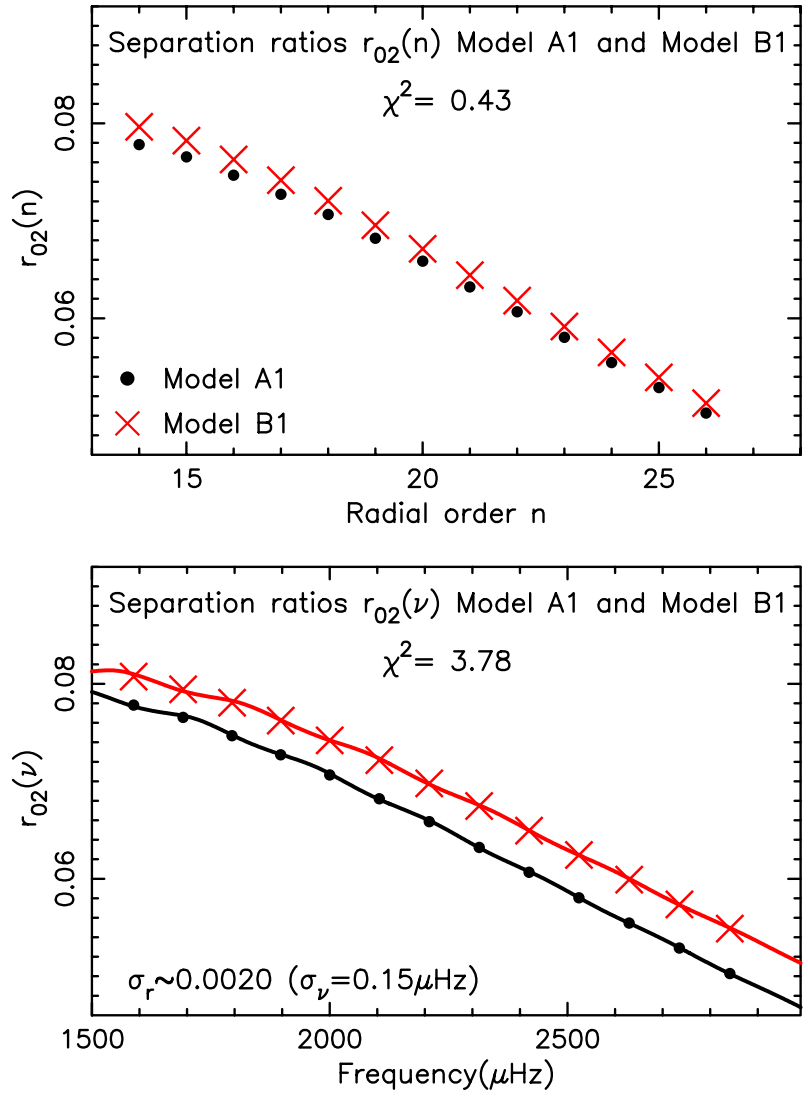

Fig. 3. Comparison of separation ratios $r_{02}$ of Model A1 and B1. Top panel: comparison at the same $n$ where the fit is good with a $\chi^{2}=0.43$; bottom panel: fit at the same frequencies which is equivalent to comparing inner phase shift differences which would agree were the two models to have the same interior structure. The continuous curves are the inner phase shift differences $\delta_{2}(v)-\delta_{0}(v)$ for the 2 models.

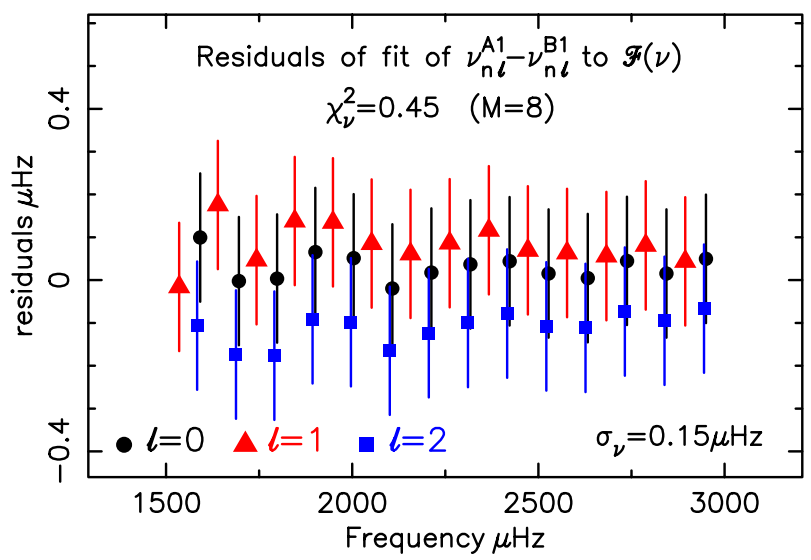

Fig. 4. Residuals of the fit of the frequency differences $v_{n \ell}^{A 1}-v_{n \ell}^{B 1}$ to a function of frequency $\mathcal{F}(v)$.

The top panel of Fig. 3 shows the ratios compared at the same $n$ values, the agreement is good with a $\chi^{2}$ of the fit $=$ 0.43 , naively suggesting a good fit between Models A1 and B1 whereas, when compared at the same frequency as in the bottom panel, the $\chi^{2}=3.78$ of the fit is very large. The continuous curves in this figure are the inner phase shift differences of the 2 models, which should agree if Model B1 is a good fit to Model A1, which it is not.

I then tested the fit of Model B1 to Model A1 using the frequency comparison algorithm defined in Eq. (18). Figure 4 
shows the residuals of the best fit of the frequency differences $v^{\mathrm{A}}-v^{\mathrm{B}}$ to a function $\mathcal{F}(v)$, which has a $\chi^{2}=0.45$, again naively suggesting a good fit of the 2 models whereas, as seen from the inner phase shift differences shown in Fig. 3, this is not the case.

I also determined the quality of fit using the phase matching algorithm described in Roxburgh \& Vorontsov (2003b) and Roxburgh (2015a); this gave a $\chi^{2}=2.35$, still very large although smaller than that from comparing the $r_{02}(v)$ of the 2 models. This is to be expected since the error estimates of frequency differences is $\sim \sqrt{2}$ larger than that on individual frequencies.

\section{Conclusions}

From a given model star (Model A) with a density profile $\rho_{\mathrm{A}}(x)$ where $x=r / R$ is the fractional radius, one can generate a two parameter family of models, the parameters being the radius $R$, and a scaling factor $\lambda$, where the density $\rho(x)$ of a member of the family is $\lambda \rho_{\mathrm{A}}(x)$. The frequencies of the models scale with $\lambda^{1 / 2}$ and the mass with $\lambda\left(R / R_{\mathrm{A}}\right)^{3}$

The family of scaled models all have the same separation ratios as a function of radial order $n$, and give an exact fit to to frequency fitting algorithm with an "offset" function of frequency determined by a best fit algorithm, even though they have a different interior density and pressure structure $\rho(r), P(r)$, demonstrating that these algorithms can give erroneous results. Model fitting techniques that compare separation ratios at the same frequency or phase matching do not suffer from this problem.

I give a specific example of a model where the scale factor and radius are such that the sound speed profile $c(x)$ is the same in both the reference model and the scaled model. I then give an example of the problem using non scaled models calculated with a stellar evolution code.

These results underline the importance when model fitting of comparing separation ratios at the same frequency, which reproduce the inner phase shift differences and therefore test how well the inner structure of a given model fits that of an observed star (cf. Roxburgh \& Vorontsov 2013). It also shows that the frequency matching algorithm with a surface offset determined by the fitting process can give erroneous results. The phase match model fitting algorithm (Roxburgh \& Vorontsov 2003b; Roxburgh 2015a) and a new algorithm using $\epsilon$ values (Roxburgh 2015b) where $v_{n \ell}=\Delta\left(n+\ell / 2+\epsilon_{n \ell}\right)$, do not suffer from these problems.

Acknowledgements. I gratefully acknowledge support from the Leverhulme Foundation under grant EM-2012-035/4 and from the UK Science and Technology Facilities Council (STFC) under grant ST/M000621/1.

\section{Appendix A: Scaling for modes of all $\ell$ values}

The full equations governing the oscillations can be written in terms of the Lagrangian pressure perturbation $\delta p$, radial perturbation $\xi$, tangential displacement $\zeta$, gravitational potential perturbation $\phi$ and angular frequency $\omega$ as (cf. Roxburgh 2008)

$$
\begin{aligned}
& \frac{\mathrm{d} \xi}{\mathrm{d} r}=-\frac{2}{r} \xi+\frac{\ell(\ell+1)}{r} \zeta-\frac{1}{\Gamma_{1} p} \delta p \\
& r \rho \omega^{2} \zeta=\delta p+\rho \xi g+\rho \phi \\
& \frac{\mathrm{d} \delta p}{\mathrm{~d} r}=\omega^{2} \rho \xi+\frac{4 g \rho \xi}{r}-\frac{\ell(\ell+1)}{r} \rho g \zeta-\rho \chi
\end{aligned}
$$

$$
\begin{aligned}
& \frac{\mathrm{d} \phi}{\mathrm{d} r}=\chi-4 \pi G \rho \xi \\
& \frac{\mathrm{d} \chi}{\mathrm{d} r}=-\frac{2}{r} \chi+\frac{\ell(\ell+1)}{r^{2}} \phi+\frac{\ell(\ell+1)}{r} 4 \pi G \rho \zeta .
\end{aligned}
$$

Dimensionally the gravitational potential $\phi$ and the derivative $\chi$ are

$$
\phi(x) \sim \frac{G M(x)}{r}, \quad \chi(x) \sim \frac{G M(x)}{r^{2}}
$$

and hence scale like

$$
\phi_{\mathrm{B}}(x)=\lambda\left(\frac{R_{\mathrm{B}}}{R_{\mathrm{A}}}\right)^{2} \phi_{\mathrm{A}}(x), \quad \chi_{\mathrm{B}}(x)=\lambda\left(\frac{R_{\mathrm{B}}}{R_{\mathrm{A}}}\right) \chi_{\mathrm{A}}(x) .
$$

Defining $y=\xi / R, z=\zeta / R$, Eqs. (A.1)-(A.5) for Model B become

$$
\frac{\mathrm{d} y}{\mathrm{~d} x}=-\frac{2}{x} y+\frac{\ell(\ell+1)}{x} z-\frac{1}{\Gamma_{1}^{\mathrm{B}}} \frac{\delta p_{\mathrm{B}}}{p_{\mathrm{B}}}
$$

$R_{\mathrm{B}}^{2} x \rho_{1} \omega_{\mathrm{B}}^{2} z=\delta p_{\mathrm{B}}+\rho_{\mathrm{B}} g_{\mathrm{B}} R_{\mathrm{B}} y+\rho_{\mathrm{B}} \phi_{\mathrm{B}}$

$$
\begin{aligned}
& \frac{1}{R_{\mathrm{B}}} \frac{\mathrm{d} \delta p_{1}}{\mathrm{~d} x}=\omega_{\mathrm{B}}^{2} \rho_{\mathrm{B}} R_{\mathrm{B}} y+\frac{4 g_{\mathrm{B}} \rho_{\mathrm{B}}}{x} y-\frac{\ell(\ell+1)}{x} \rho_{\mathrm{B}} g_{\mathrm{B}} z-\rho_{\mathrm{B}} \chi_{\mathrm{B}}(A) 1 \\
& \frac{\mathrm{d} \phi_{\mathrm{B}}}{\mathrm{d} x}=R_{\mathrm{B}} \chi_{\mathrm{B}}-4 \pi G \rho_{\mathrm{B}} R_{\mathrm{B}}^{2} y \\
& \frac{\mathrm{d} \chi_{\mathrm{B}}}{\mathrm{d} x}=-\frac{2}{x} \chi_{\mathrm{B}}+\frac{\ell(\ell+1)}{R_{\mathrm{B}} x^{2}} \phi_{\mathrm{B}}+\frac{\ell(\ell+1)}{x} 4 \pi G R_{\mathrm{B}} \rho_{\mathrm{B}} z
\end{aligned}
$$

Substituting the scaling relations as in Eqs. (11) and (A.7) into Eqs. (A.8)-(A.12) one obtains the same equations for the reference model variables with subscript A provided $\omega_{\mathrm{B}}^{2}=\lambda \omega_{\mathrm{A}}^{2}$, exactly as in the analysis for radial $\ell=0$ modes.

Hence for any $R_{\mathrm{B}}$ and $\lambda$ we have the scaling relations

$$
\begin{aligned}
& \rho_{\mathrm{B}}(x)=\lambda \rho_{\mathrm{A}}(x), \quad M_{\mathrm{B}}=\lambda \frac{R_{\mathrm{B}}^{3}}{R_{\mathrm{A}}^{3}} M_{\mathrm{A}}, \quad v_{n \ell}^{\mathrm{B}}=\lambda^{1 / 2} v_{n \ell}^{\mathrm{A}} \\
& P_{\mathrm{B}}(x)=\lambda^{2}\left(\frac{R_{\mathrm{B}}}{R_{\mathrm{A}}}\right)^{2} P_{\mathrm{A}}(x), \quad g_{\mathrm{B}}(x)=\lambda\left(\frac{R_{\mathrm{B}}}{R_{\mathrm{A}}}\right) g_{\mathrm{A}}(x) .
\end{aligned}
$$

\section{References}

Angulo, C., \& the NACRE consortium 1999, Nucl. Phys. A, 656, 3 Christensen-Dalsgaard, J., Dappen, W., Ajukou, S. V., et al. 1996, Science, 272, 1286

Ferguson, J. W., Alexander, D. R., Allard, D. T., et al. 2005, ApJ, 623, 585 Grevesse, N., \& Sauval, A. J. 1998, Space Sci. Rev., 85, 161

Iglesias, C. A., \& Rogers, F. J. 1996, ApJ, 464, 943

Kjeldsen, H., Bedding, T. R., \& Christensen-Dalsgaard, J. 2008, ApJ, 683, L175

Rogers, F. J., \& Nayfonov, A. 2002, ApJ, 576, 1064

Roxburgh, I. W. 2008, Ap\&SS, 316, 141

Roxburgh, I. W. 2009, Presentation at meetings: Synergies between solar and stellar modelling, Rome, June 22-26, New Insights into the Sun, the potential of a new generation of solar-stellar diagnostics, Ponte de Lima, Portugal, September 16-18

Roxburgh, I. W. 2015a, A\&A, 574, A45

Roxburgh, I. W. 2015b, A\&A, in press, DOI: 10.1051/0004-6361/201526593

Roxburgh, I. W., \& Vorontsov S. V. 2003a, A\&A, 411, 215

Roxburgh, I. W., \& Vorontsov S. V. 2003b, Ap\&SS, 284, 187

Roxburgh, I. W., \& Vorontsov S. V. 2013, A\&A, 560, A2 